Article

\title{
Examining the Feasibility of the Sturm-Liouville Theory for Ross Recovery
}

\author{
Shinmi Ahn ${ }^{1}$ and Hyungbin Park ${ }^{2, *}$ \\ 1 Graduate School, Kyung Hee University, 6, Kyungheedae-ro, Dongdaemun-gu, Seoul 02453, Korea; \\ pletnev830@gmail.com \\ 2 Department of Mathematical Sciences and RIMS, Seoul National University, 1, Gwanak-ro, Gwanak-gu, \\ Seoul 08826, Korea \\ * Correspondence: hyungbin2015@gmail.com or hyungbin@snu.ac.kr
}

Received: 6 February 2020; Accepted: 5 April 2020; Published: 9 April 2020

check for updates

\begin{abstract}
Recent studies have suggested that it is feasible to recover a physical measure from a risk-neutral measure. Given a market state variable modeled as a Markov process, the key concept is to extract a unique positive eigenfunction of the generator of the Markov process. In this work, the feasibility of this recovery theory is examined. We prove that, under a restrictive integrability condition, recovery is feasible if and only if both endpoints of the state variable are limit-point. Several examples with explicit positive eigenfunctions are considered. However, in general, a physical measure cannot be recovered from a risk-neutral measure. We provide a financial and mathematical rationale for such recovery failure.
\end{abstract}

Keywords: Ross recovery; Sturm-Liouville theory; physical measure; risk-neutral measure; pricing kernel; Markov process

\section{Introduction}

This study is aimed at examining the relationship between physical measures and risk-neutral measures. A physical measure describes the actual probability distribution of asset prices, whereas a risk-neutral measure is a theoretical measure to determine the prices of financial derivatives. It is commonly accepted that a risk-neutral measure cannot be used to infer a physical measure. However, Ross [1] and Carr and Yu [2] have recently argued that, given strong assumptions, a risk-neutral measure can be used to determine a physical measure. In this work, we review the methodologies adopted by these authors and give a further improved analysis.

The main arguments presented in the above-mentioned articles are as follows: Ross [1] considered an economy with a state variable modeled as a discrete-time finite-state Markov process in its steady-state probability distribution. The author exploited the concept of the utility of a representative agent and analyzed the optimizing behavior of the representative agent. Ross proved that the physical measure can be determined in terms of an eigenvalue and eigenvector of the generator matrix of the Markov process. Carr and Yu [2] presented a similar argument, which applies to a state variable modeled as a continuous-time univariate Markov diffusion process restricted to a bounded interval. The authors employed the concept of Long's numeraire portfolio driven by the state variable, instead of the concept of the utility of a representative agent. They demonstrated that the physical measure can be determined in terms of an eigenvalue and eigenfunction of the infinitesimal generator of the Markov diffusion process. Both these arguments involved deep economic and financial concepts and mathematical analyses.

A crucial step performed in the above-mentioned studies is the uniqueness (up to a scalar multiple) of the positive eigenvector or eigenfunction of the generator of the Markov process or Markov diffusion, 
respectively. This generator may be a stochastic matrix, as considered by Ross [1], or a second-order differential operator, as considered by Carr and Yu [2]. Ross employed the Perron-Frobenius theorem to extract the unique positive eigenvector. Along the same lines, Carr and Yu exploited the regular Sturm-Liouville (SL) theory to extract the unique positive eigenfunction. Finally, using this unique eigenvector or eigenfunction, a physical measure could be determined from the risk-neutral measure.

Ross recovery has been studied by many authors from diverse economic viewpoints. Borovička et al. [3] extracted a positive martingale component of the stochastic discount factor process by using the Perron-Frobenius theory, which could be used to recover a probability measure that absorbs the long-term risk adjustments. Jensen et al. [4] characterized the cases in which a physical measure could be recovered from the risk-neutral measure for several future time periods. The authors concluded that recovery is feasible if the number of maturities with observable prices is higher than the number of states of the economy. Park [5] provided a sufficient condition that recovery is possible in the continuous-time model when the state variable is transient under the physical measure. He proved that one-parameter recovery is possible if the state variable is not attracted to the left (or right) boundary. Qin and Linetsky [6] demonstrated that a physical measure can be recovered from a risk-neutral measure if the state variable is recurrent under the physical measure. Walden [7] analyzed the problem of recovering a physical measure from the observed option prices when the state variable is modeled as an unbounded diffusion process. He derived a necessary and sufficient condition for recovery in terms of the unbounded diffusion process.

Furthermore, several methods have been developed to implement the Ross recovery theorem for empirical analyses. Audrino et al. [8] conducted an empirical analysis of the Ross recovery theorem. The researchers investigated whether the recovery yields predictive information beyond what can be gleaned from the risk-neutral measure and concluded that market timing strategies based on the recovered measure significantly outperform their risk-neutral counterparts. Bakshi et al. [9] studied the reliability of the Ross recovery theorem. It was observed that the empirical results undermine the implications of the recovery theorem, and the authors rejected the assumption that the reciprocal of the pricing kernel is transition-independent. Dillschneider and Maurer [10] generalized the Ross recovery theorem to continuous-state spaces. Furthermore, building on the theoretical results, the researchers proposed a non-parametric approach to empirically estimate the recovered physical measure. Flint and Maré [11] investigated a regularization methodology for extracting a physical measure and implemented this method on a history of stochastic volatility inspired surfaces. Kiriu and Hibiki [12] presented a method to accurately estimate a physical measure by using the Ross recovery theorem and demonstrated the effectiveness of this approach by performing a numerical analysis with hypothetical data.

In this article, we raise questions related to the economic/financial arguments in the existing studies. All the past studies of Ross recovery have employed the market models developed by either Ross [1] or Carr and Yu [2]. Under these models, we agree that the risk-neutral measure contains some information regarding the physical measure; however, we do not believe that the physical measure can be uniquely determined by the risk-neutral measure. In the Ross model, the state variable has a finite-state space. This assumption is unrealistic as the possible stock prices are finite and, thus, once a stock price hits a maximum value, it must decrease. Similarly, once a stock price hits a minimum value, it must increase. In contrast, in an actual financial market, the stock prices can always increase or decrease further. We show, in Section 5.2, that if the state variable is infinite, a physical measure cannot be uniquely determined by the risk-neutral measure. A similar issue arises in the model of Carr and Yu [2], who use the regular Sturm-Liouville (SL) theory to extract a unique eigenfunction $h$ (Observation 1).

The remaining paper is structured as follows: The original argument of the recovery theorem presented by Ross [1] is described in Section 2. Section 3 demonstrates the regular SL theory as a mathematical tool and later describes the continuous-time model developed by Carr and Yu [2]. The necessary details of the two papers are recalled to establish our argument more effectively. Section 4 
discusses the economic and financial limits of the recovery arguments presented by Ross [1] and Carr and $\mathrm{Yu}$ [2]. Furthermore, we provide a slightly modified version of the Ross recovery to overcome these limits, and several examples with explicit solutions are studied. In Section 5, we discuss the impossibility of the Ross recovery theory in general circumstances. The final section summarizes the key findings of this work.

\section{Original Ross Recovery Theorem}

This section provides a brief review of the recovery theorem provided by Ross [1]. Ross suggested that a physical measure can be recovered from a risk-neutral measure under a discrete-time and finite-state economy. Using the Perron-Frobenius theorem (Appendix A), he concluded that only one possible physical measure can be chosen. We examine that this argument leads to an eigenvalue problem for a square matrix which satisfies the hypotheses of the Perron-Frobenius theorem. The approach outlined in this section is standard in financial economics, and Chapter 4 of [13] is a useful reference. The terminologies and methodologies referred to are from Carr and $\mathrm{Yu}$ [2].

The assumptions of the original Ross recovery theorem are as follows: Let $n \in \mathbb{N}$ be the number of possible states of the market. The time periods are indexed by integers $t \in \mathbb{T}:=\{0,1, \cdots, T\}$, and the state variable at time $t$ is denoted as $X_{t}$ with $X_{t} \in\{1,2, \ldots, n\}$. We ignore the distinction between $X_{t} \in\{1,2, \ldots, n\}$ being the state or the label of the state. The physical transition probabilities and the risk-neutral transition probabilities are denoted by $f_{i j}$ and $p_{i j}$, respectively, and these transition probabilities are independent of the time $t$. In other words, $f_{i j}=\operatorname{Prob}\left(X_{t+1}=j \mid X_{t}=i\right)$ and (if $\left.X_{t}=i\right) p_{i j}$ is the market price at period $t$ of a contract that pays one unit of currency at the next time period if $X_{t+1}=j$, and is nothing if $X_{t+1} \neq j$. As is well-known, $p_{i j}$ is the Arrow-Debreu state price. These probabilities are positive; that is, $f_{i j}>0$ and $p_{i j}>0$ for all $1 \leq i, j \leq n$.

Ross also assumed that there exists a representative agent in the market, which satisfies the following conditions:

(i) The consumption $C_{t}$ at time $t$ of the representative agent depends on the time and the state variable; that is, $C_{t}=C\left(X_{t}, t\right)$ for some function $C(\cdot, \cdot)$.

(ii) The utility of the representative agent is dependent on the consumption and is independent of the time; hence, the utility at time $t$ can be expressed by $U\left(C_{t}\right)$ for the utility function $U(\cdot)$.

(iii) The short-interest rate at time $t$, denoted as $r_{t}$, is determined by the state variable $X_{t}$; that is, $r_{t}=r\left(X_{t}\right)$ for some function $r(\cdot)$.

We consider the representative agent in one time period (e.g., between $t=0$ and $t=1$ ), as the state variable $\left(X_{t}\right)_{t \in \mathbb{T}}$ is time-homogeneous. At $t=0$, we assume that the state is $X_{0}=i$, and the representative agent has an initial endowment $K>0$. The agent consumes $c_{i 0}=C(i, 0)$ at $t=0$, and the remainder is invested in the Arrow-Debreu securities:

$$
c_{i 0}+\sum_{j=1}^{N} c_{j 1} p_{i j}=K,
$$

where $c_{j 1}$ denotes the amount of the $j^{\text {th }}$ h security invested. At $t=1$, if the state is $j$, the agent earns $c_{j 1}$. The agent consumes $c_{j 1}$ and obtains the utility $U\left(c_{j 1}\right)$. Through these consumptions, the agent wants to maximize

$$
U\left(c_{i 0}\right)+\delta \sum_{j=1}^{N} U\left(c_{j 1}\right) f_{i j},
$$

where $0<\delta<1$ is a discount factor. The agent confronts this maximization problem under the budget constraint given in Equation (1). 
To solve this, we apply the Lagrangian method. We define

$$
\mathcal{L}=U\left(c_{i 0}\right)+\delta \sum_{j=1}^{N} U\left(c_{j 1}\right) f_{i j}-\lambda\left(c_{i 0}+\sum_{j=1}^{N} c_{j 1} p_{i j}-K\right) .
$$

The first-order condition with respect to $c_{i 0}$ and $c_{j 1}$ yields

$$
\left\{\begin{array}{l}
U^{\prime}\left(c_{i 0}\right)-\lambda=0 \\
\delta U^{\prime}\left(c_{j 1}\right) f_{i j}-\lambda p_{i j}=0 .
\end{array}\right.
$$

Eliminating $\lambda$, we conclude that

$$
f_{i j}=\frac{1}{\delta} \frac{U^{\prime}\left(c_{i 0}\right)}{U^{\prime}\left(c_{j 1}\right)} p_{i j}
$$

Ross assumed that the market is at a steady state, implying that the consumption depends on the state, but not on the time; that is, $c_{j 0}=c_{j 1}$ for all $j$. Hence, denoting $c_{j}=c_{j 0}=c_{j 1}$, we have

$$
f_{i j}=\frac{1}{\delta} \frac{U^{\prime}\left(c_{i}\right)}{U^{\prime}\left(c_{j}\right)} p_{i j}
$$

We sum over the index $j$ and use $\sum_{j=1}^{N} f_{i j}=1$. After some manipulation and considering $\pi_{j}=\frac{1}{U^{\prime}\left(c_{j}\right)}$, it follows that

$$
\sum_{j=1}^{N} p_{i j} \pi_{j}=\delta \pi_{i}
$$

In matrix notation, by defining $P=\left(p_{i j}\right), \pi=\left(\pi_{j}\right)$, the above equation becomes

$$
P \pi=\delta \pi
$$

Here, the matrix $P$ is known; however, $\pi$ and $\delta$ are unknown. The discount factor $\delta$ and all entries of $\pi$ should be positive. The Perron-Frobenius theorem (Appendix A) states that there exist a unique positive number $\delta$ and a unique vector $\pi$ with positive entries up to constant multiples satisfying Equation (4). Once $\delta$ and $\pi$ are obtained, we recover $\left(f_{i j}\right)_{0 \leq i, j \leq n}$ using Equation (3).

\section{Model Proposed by Carr and Yu}

Carr and $\mathrm{Yu}$ [2] developed the Ross recovery theorem in two significant aspects. One aspect is that the model assumes a continuous-time and continuum-state economy, and the other aspect is that the model exploits the martingale approach, instead of using the concept of a representative agent. In this section, we review the model of Carr and Yu and describe their methodology to recover the physical measure.

\subsection{Principal Pair}

This section demonstrates how the market was modeled by Carr and Yu [2]. The model setup described in this section may appear simpler than the original setup of Carr and Yu. However, they are equivalent. This simplified setup is referred to from Park [5]. Let $(\Omega, \mathcal{F}, \mathbb{P})$ be a probability space having a Brownian motion $B=\left(B_{t}\right)_{t \geq 0}$, and let $\left(\mathcal{F}_{t}\right)_{t \geq 0}$ be the completed filtration generated by $B$. The measure $\mathbb{P}$ is referred to as the physical measure and is assumed to be unknown. A money market account is a progressively measurable positive process, denoted by $G=\left(G_{t}\right)_{t \geq 0}$. Let $\mathbb{Q}$ be a risk-neutral measure; that is, $\mathbb{P}$ and $\mathbb{Q}$ are equivalent and each asset discounted by $G$ is a $\mathbb{Q}$-martingale. Define $\Sigma=\left(\Sigma_{t}\right)_{t \geq 0}$ as a continuous modification of the process $\left(\mathbb{E}^{\mathbb{P}}\left[\frac{d \mathbb{Q}}{d \mathbb{P}} \mid \mathcal{F}_{t}\right]\right)_{t \geq 0}$. According to the martingale representation theorem, there exists a progressively measurable process $\rho=\left(\rho_{t}\right)_{t \geq 0}$ such 
that $\rho$ is locally square-integrable and the stochastic differential equation (SDE) $d \Sigma=-\rho_{t} \Sigma_{t} d B_{t}$ holds. According to the Girsanov theorem, a process

$$
W=\left(W_{t}\right)_{t \geq 0}=\left(B_{t}+\int_{0}^{t} \rho_{s} d s\right)_{t \geq 0}
$$

is a $\mathbb{Q}$-Brownian motion. Here, the risk-neutral $\mathbb{Q}$ and the Brownian motion $W$ are assumed to be known. We define the reciprocal of the pricing kernel as

$$
L=\left(L_{t}\right)_{t \geq 0}=\left(G_{t} / \Sigma_{t}\right)_{t \geq 0} .
$$

Assumption 1. In the market, there exists a state variable $X=\left(X_{t}\right)_{t \geq 0}$ with state space $I:=(a, b)$ for $-\infty \leq a<b \leq \infty$. The process $X$ is a time-homogeneous Markov diffusion satisfying

$$
d X_{t}=k\left(X_{t}\right) d t+\sigma\left(X_{t}\right) d W_{t}, X_{0}=\xi .
$$

The functions $k$ and $\sigma$ are continuous on $I$, and $\sigma$ is positive.

Assumption 2. The money market account is determined by the state variable. More precisely, there exists a positive continuous function $r$ on I such that

$$
G_{t}=e^{\int_{0}^{t} r\left(X_{s}\right) d s}, t \geq 0 .
$$

Assumption 3. Assume that the reciprocal of the pricing kernel $L$ is transition-independent; that is, there exists a positive function $h \in C^{2}(I)$ and a real number $\lambda$ such that

$$
L_{t}=e^{\lambda t} \frac{h\left(X_{t}\right)}{h\left(X_{0}\right)}, t \geq 0
$$

In this case, it is considered that $\lambda, h$, and $(\lambda, h)$ are the principal value, principal function, and principal pair of the market, respectively.

The basis of the recovery theory is finding the principal pair $(\lambda, h)$ and then obtaining the physical measure $\mathbb{P}$ by setting the Radon-Nikodym derivative

$$
\left.\frac{d \mathbb{P}}{d \mathbb{Q}}\right|_{\mathcal{F}_{t}}=\frac{1}{\Sigma_{t}}=e^{\lambda t-\int_{0}^{t} r\left(X_{s}\right) d s} \frac{h\left(X_{t}\right)}{h\left(X_{0}\right)} .
$$

According to the Girsanov theorem, the state variable $X$ follows

$$
d X_{t}=\left(k+\sigma^{2} h^{\prime} / h\right)\left(X_{t}\right) d t+\sigma\left(X_{t}\right) d B_{t}
$$

under the physical measure.

Under Assumptions 1-3, we show that the problem of finding the principal pair can be transformed into a second-order differential equation problem. Applying the Itô formula to Equation (5) yields

$$
\frac{d L_{t}}{L_{t}}=r\left(X_{t}\right) d t+\rho_{t} d W_{t}
$$

Furthermore, by Equation (6), we have

$$
\frac{d L_{t}}{L_{t}}=\left(\lambda+\frac{1}{2}\left(\sigma^{2} h^{\prime \prime} / h\right)\left(X_{t}\right)+\left(k h^{\prime} / h\right)\left(X_{t}\right)\right) d t+\left(\sigma h^{\prime} / h\right)\left(X_{t}\right) d W_{t} .
$$


By comparing the two SDEs, it follows that

$$
\left\{\begin{array}{l}
\mathcal{L} h=-\lambda h \text { on } I \\
\rho=\left(\sigma h^{\prime} / h\right)(X)
\end{array},\right.
$$

where $\mathcal{L}$ is an operator defined on $C^{2}(I)$ by

$$
\mathcal{L} h(x)=\frac{1}{2} \sigma^{2}(x) h^{\prime \prime}(x)+k(x) h^{\prime}(x)-r(x) h(x)
$$

for $x \in I$. Finally, we have the following theorem.

Theorem 1. Under Assumptions 1-3, let $(\lambda, h)$ be the principal pair of the market so that the process $M^{h}:=\left(e^{\lambda t-\int_{0}^{t} r\left(X_{s}\right) d s} h\left(X_{t}\right) / h\left(X_{0}\right)\right)_{t \geq 0}$ in Equation (7) is a martingale under the measure $\mathbb{Q}$. Then the pair $(\lambda, h)$ satisfies

$$
\mathcal{L} h=-\lambda h \text { on } I .
$$

Conversely, if a pair $(\lambda, h)$ with $\lambda \in \mathbb{R}$ and $h \in C^{2}(I)$ satisfies Equation (9), then the process $M^{h}$ is a local martingale under the measure $\mathbb{Q}$.

Remark 1. For an eigenvalue $\lambda \in \mathbb{R}$ and a positive eigenfunction $h \in C^{2}(I)$ of Equation (9), the process $M^{h}$ is a local martingale under the measure $\mathbb{Q}$. However, to ensure that the change of measure is well-defined (Equations (5) and (6)), one needs to ensure that this process $M^{h}$ is a martingale. There is a useful criterion (Theorem 5.1 .8 of [14]) to check whether this process is a martingale or not. Let $x_{0} \in I=(a, b)$. The process $M^{h}$ is a martingale if and only if

$$
\begin{aligned}
& \int_{a}^{x_{0}} \frac{1}{h^{2}(x)} e^{-\int_{x_{0}}^{x} \frac{2 k(s)}{\sigma^{2}(s)} d s} \int_{x}^{x_{0}} \frac{h^{2}(y)}{\sigma^{2}(y)} e^{\int_{x_{0}}^{y} \frac{2 k(s)}{\sigma^{2}(s)} d s} d y d x=\infty, \\
& \int_{x_{0}}^{b} \frac{1}{h^{2}(x)} e^{-\int_{x_{0}}^{x} \frac{2 k(s)}{\sigma^{2}(s)} d s} \int_{x}^{x_{0}} \frac{h^{2}(y)}{\sigma^{2}(y)} e^{\int_{x_{0}}^{y} \frac{2 k(s)}{\sigma^{2}(s)} d s} d y d x=\infty .
\end{aligned}
$$

\subsection{Regular Sturm-Liouville Theory}

To find the principal pair, Carr and Yu [2] employed the regular SL theory. The mathematical tool corresponding to the Perron-Frobenius theorem in the Ross model is the regular SL theory in the model of Carr and Yu. Recall that the principal pair $(\lambda, h)$ satisfies $\mathcal{L} h=-\lambda h$ on $I$ presented in Equation (9). This is an eigenvalue-eigenfunction problem, as the unknown is a pair $(\lambda, h)$.

Equation (9) can be transformed as follows: Define

$$
\begin{aligned}
w(x) & =\frac{2}{\sigma^{2}(x)} e^{\int_{x_{0}}^{x} \frac{2 k(z)}{\sigma^{2}(z)} d z}, \\
p(x) & =\frac{1}{2} \sigma^{2}(x) w(x), \\
q(x) & =r(x) w(x)
\end{aligned}
$$

for any reference point $x_{0} \in I$. Let $A$ be an operator, given as

$$
A h=-\left(p h^{\prime}\right)^{\prime}+q h
$$

on the domain

$$
L_{w}^{2}(I)=\left\{u \in C^{2}(I) \mid \int_{I} u^{2}(y) w(y) d y<\infty\right\} .
$$


The function $w$ is known as the weight function. Note that $p, q$, and $w$ are continuous, and $p>0$ and $w>0$ on $I$. It can be easily shown that Equation (9) is equivalent to

$$
A h=\lambda w h \quad \text { on } I .
$$

The operator $A$ and Equation (12) are called the SL operator and SL equation, respectively.

A regular SL operator has advantageous properties. We say that an SL operator $A$ (or an SL equation $A h=\lambda w h$ ) is regular if

$$
\frac{1}{p}, q, w \in L^{1}(I)
$$

and is singular if it is not regular. A regular equation $A h=\lambda w h$ on $L_{w}^{2}(I)$ with the boundary conditions of the form

$$
\begin{cases}\alpha_{1} h(a)+\alpha_{2} h^{\prime}(a)=0, & \alpha_{1}^{2}+\alpha_{2}^{2} \neq 0 \\ \beta_{1} h(b)+\beta_{2} h^{\prime}(b)=0, & \beta_{1}^{2}+\beta_{2}^{2} \neq 0\end{cases}
$$

has advantageous properties:

(i) The spectrum consists of eigenvalues that are discrete, real, bounded below, and have no cluster point. We can denote these values as

$$
\lambda_{0}<\lambda_{1}<\lambda_{2}<\cdots
$$

(ii) An eigenfunction $h_{n} \in L_{w}^{2}(I)$ corresponding to the eigenvalue $\lambda_{n}$ has $n$ zeros on the domain $I$. Therefore, $h_{0}$ has no zeros and can be taken as positive.

\subsection{Methodology Employed by Carr and $Y u$}

We impose an additional assumption on the principal function $h$ to apply the SL theory. Recall that $L_{w}^{2}(I)$ is the space defined in Equation (11).

Assumption 4. The principal function $h$ is in $L_{w}^{2}(I)$.

The methodology employed by Carr and $\mathrm{Yu}$ is as follows: Suppose that the operator $A$ in Equation (12) is regular, and a suitable boundary condition (Equation (13)) is given on a bounded domain $I=(a, b)$. Then, there exists a smallest eigenvalue $\lambda_{0}$ of the operator $A$, as stated in Equation (14). We denote the corresponding eigenfunction by $h_{0}(\cdot)$ (with the normalization $h_{0}\left(X_{0}\right)=1$ ). According to the regular SL theorem, $h_{0}(\cdot)$ is the unique positive solution (up to constant multiples) such that Equation (12) holds. Thus, $\left(\lambda_{0}, h_{0}\right)$ is the only candidate for the principal pair, and it follows that $L_{t}=$ $e^{\lambda_{0} t} h_{0}\left(X_{t}\right), t \geq 0$. In conclusion, one can recover a physical measure from the risk-neutral measure.

However, the authors cannot see any reasonable arguments for the assumptions of this approach:

Observation 1. Consider the methodology employed by Carr and $Y u$.

(1) There is no economic or financial rationale to believe that $h$ lies in $L_{w}^{2}(I)$ (Assumption 4).

(2) The SL equations are not regular for most financially meaningful models.

(3) There are no economic or financial reasons to impose such a boundary condition (Equation (13)).

The rest of this paper corresponds to our investigation of overcoming these assumptions. It is relatively straightforward to overcome (2), by applying the singular SL theory. Furthermore, (3) can be overcome by considering a limit-cycle/limit-point boundary classification. It is later demonstrated that no boundary conditions are required if and only if both the boundary endpoints of the domain are limit-point. Section 4 presents the method applied to overcome (2) and (3). Unfortunately, it appears that (1) cannot be overcome; we explore why it fails in Section 5. 


\section{Singular Sturm-Liouville Theory}

This section develops the Ross recovery theorem in the context of the model of Carr and Yu. First, the concept of the limit-cycle (LC)/limit-point (LP) classification of the endpoints is introduced. Subsequently, we prove that recovery is feasible if and only if both the endpoints are LP, which is one of main findings of the present work. Finally, explicit recovery formulas are provided for several cases, including Brownian motion, geometric Brownian motion, the Cox-Ingersoll-Ross (CIR) process, and the constant elasticity of variance $(\mathrm{CEV})$ process.

\subsection{LC/LP Dichotomy}

We investigate the notion of the LC/LP classification. Recall the SL equation

$$
A h=-\left(p h^{\prime}\right)^{\prime}+q h=\lambda w h
$$

on $I=(a, b)$. We say that an endpoint $a$ is

(i) an LC, if all solutions of the SL equation are in $L_{w}^{2}((a, c))$ for some (and, hence, any) $c \in(a, b)$; and

(ii) an LP, if it is not an LC.

Similar definitions can be provided for $b$. It is well-known that the LC/LP classification is independent of $\lambda \in \mathbb{R}$ (Section 7.2 and the last paragraph on page 145 in [15]).

Now, we state a criterion for the LC/LP classification. The following proposition is simple, but is particularly useful for our analysis. For the proof, see ([15] Theorem 7.4.1).

Proposition 1. An endpoint $a$ is $L P$ if $q \geq k w$ on $(a, c)$ and $w \notin L^{1}(a, c)$ for some $k \in \mathbb{R}$ and $c \in(a, b)$. A similar criterion holds for $b$.

\subsection{Recovery Theory}

In this section, we investigate a necessary and sufficient condition under which a physical measure can be recovered from a risk-neutral measure. The condition is stated in the context of the LC/LP classification.

Definition 1. Under Assumptions 1-4, we say that a physical measure can be recovered from a risk-neutral measure if the SL Equation (9) (or, equivalently, Equation (12)) has a unique (up to constant multiples) positive eigenfunction in $L_{w}^{2}(I)$. In this case, we say that recovery is feasible.

The main finding of this work pertains to Theorem 3, which can be easily obtained from Theorem 2. Both Theorems 2 and 3 are original results of this paper.

Theorem 2. Assume that the SL Equation (12) has at least one positive eigenfunction $h \in L_{w}^{2}(I)$.

(i) If at least one endpoint of I is $L C$, then there is an infinite number of positive eigenfunctions in $L_{w}^{2}(I)$.

(ii) If both endpoints of I are LP, then there is a unique positive eigenfunction (up to constant multiples) in $L_{w}^{2}(I)$.

Proof. As the SL operator $A$ is assumed to have a positive eigenfunction, every self-adjoint extension of $A$ is bounded below ([16] Theorem 12 (ii)). Each self-adjoint extension of $A$ has a unique positive eigenfunction (up to constant multiples) ([16] Theorems 14 and 15). If at least one endpoint of $I$ is LC, then there is an infinite number of extensions depending on the boundary conditions ([15] Theorem 10.4.1, Remarks 10.4.2 and 10.4.3). Thus, the SL operator $A$ has an infinite number of positive eigenfunctions. In contrast, if both the endpoints are LP, then there exists only one self-adjoint extension. Thus, $A$ has a unique positive eigenfunction (up to constant multiples). 
Theorem 3. Under Assumptions 1-4, a physical measure can be recovered from a risk-neutral measure if and only if the SL Equation (9) has both LP endpoints.

If the SL equation has both LP endpoints, the unique positive eigenfunction $h$ (up to constant multiples) is the principal function. Let $\lambda$ be the eigenvalue corresponding to $h$, then

$$
L_{t}=e^{\lambda t} h\left(X_{t}\right), t \geq 0
$$

with normalization $h\left(X_{0}\right)=1$.

Remark 2. When the SL equation has both LP endpoints, the process $M^{h}$ in Theorem 1 induced by the unique positive eigenfunction $h$ (up to constant multiples) and the corresponding eigenvalue $\lambda$ is a local martingale under the measure $\mathbb{Q}$, but may not be a true martingale. As discussed in Remark 1, the true martingale property is necessary to ensure that the change of measure in Equations (5) and (6) is well-defined. We emphasize that the true martingale property is not automatically implied by Theorems 2 and 3. For all examples in Section 4.3, this process $M^{h}$ is a true martingale, and this can be checked case by case using the criterion in Equation (10).

\subsection{Examples}

In this section, we provide some examples of the recovery theory with closed-form solutions. In particular, we consider four processes: Brownian motion, geometric Brownian motion, the CIR process, and the CEV process.

\subsubsection{Brownian Motion}

Suppose that the state variable $X$ follows a scaled Brownian motion:

$$
X_{t}:=\sigma W_{t}, t \geq 0
$$

for $\sigma>0$, and the short rate is $r\left(X_{t}\right)=\frac{1}{2} X_{t}^{2}$ at time $t \geq 0$. The range of the state variable is $I=(-\infty, \infty)$. Then, Equation (9) becomes

$$
\frac{1}{2} \sigma^{2} h^{\prime \prime}(x)-\frac{1}{2} x^{2} h(x)=-\lambda h(x)
$$

for $x \in \mathbb{R}$. Both endpoints $-\infty$ and $\infty$ are LP, by Proposition 1. It can be verified that $h_{0}(x)=e^{-\frac{1}{2 \sigma} x^{2}}$ is a unique positive eigenfunction (up to constant multiples), and its eigenvalue is $\lambda_{0}=\frac{\sigma}{2}$. Then, the reciprocal of the pricing kernel is

$$
L_{t}=e^{\frac{1}{2} \sigma t-\frac{1}{2 \sigma} X_{t}^{2}}
$$

and the state variable follows

$$
d X_{t}=-\sigma X_{t} d t+\sigma d B_{t}
$$

under the physical measure. The dynamics of the short rate $r_{t}=r\left(X_{t}\right), t \geq 0$ are

$$
\begin{aligned}
d r_{t} & =\frac{\sigma^{2}}{2} d t+\sigma X_{t} d W_{t} \\
& =\left(\frac{\sigma^{2}}{2}-2 \sigma r_{t}\right) d t+\sigma X_{t} d B_{t} .
\end{aligned}
$$

Defining $\tilde{B}=\left(\tilde{B}_{t}\right)_{t \geq 0}=\left(\int_{0}^{t} \operatorname{sign}\left(X_{s}\right) d B_{s}\right)_{t \geq 0}$, the process $\tilde{B}$ is a Brownian motion where $\operatorname{sign}(x)=1$ if $x \geq 0$ and $\operatorname{sign}(x)=-1$ if $x<0$. Then,

$$
d r_{t}=\left(\frac{\sigma^{2}}{2}-2 \sigma r_{t}\right) d t+\sigma \sqrt{2 r_{t}} d \tilde{B}_{t}
$$

which means that the short rate is a CIR process under the physical measure. 


\subsubsection{Geometric Brownian Motion}

Consider a state variable $X$ which follows the geometric Brownian motion

$$
d X_{t}=\mu X_{t} d t+\sigma X_{t} d W_{t}, X_{0}=1
$$

for $\mu \neq \frac{1}{2} \sigma^{2}$. The range of the state variable is $I=(0, \infty)$. We define $\alpha=1-\frac{2 \mu}{\sigma^{2}}$ and assume that the short rate function is $r(x)=\frac{1}{2} \alpha^{2} \sigma^{2}\left(\alpha \ln x+\frac{1}{2}\right)^{2}$ for $x \in I$. Equation (9) becomes

$$
\frac{1}{2} \sigma^{2} x^{2} h^{\prime \prime}(x)+\mu x h^{\prime}(x)-r(x) h(x)=-\lambda h(x)
$$

for $x \in I$. Both endpoints 0 and $\infty$ are LP by Proposition 1 . It can be shown that $h_{0}(x)=e^{-\frac{1}{2} \alpha^{2}(\ln x)^{2}}$ is a unique positive eigenfunction (up to constant multiples), and the eigenvalue is $\lambda_{0}=\frac{5}{8} \alpha^{2} \sigma^{2}$. Subsequently, the reciprocal of the pricing kernel is

$$
L_{t}=e^{\frac{5}{8} \alpha^{2} \sigma^{2} t-\frac{1}{2} \alpha^{2}\left(\ln X_{t}\right)^{2}}, t \geq 0
$$

and the state variable follows

$$
d X_{t}=\left(\mu-\alpha^{2} \sigma^{2} \ln X_{t}\right) X_{t} d t+\sigma X_{t} d B_{t}
$$

under the physical measure. The dynamics of the short rate $r_{t}=r\left(X_{t}\right), t \geq 0$ are

$$
\begin{aligned}
d r_{t} & =\frac{1}{4} \alpha^{4} \sigma^{4}\left(1-2 \alpha \ln X_{t}\right) d t+\alpha^{3} \sigma^{3}\left(\alpha \ln X_{t}+\frac{1}{2}\right) d W_{t} \\
& =\alpha^{4} \sigma^{4}\left(\frac{1}{4}-\alpha \ln X_{t}-\alpha^{2}\left(\ln X_{t}\right)^{2}\right) d t+\alpha^{3} \sigma^{3}\left(\alpha \ln X_{t}+\frac{1}{2}\right) d B_{t}
\end{aligned}
$$

\subsubsection{CIR Process I}

Consider the state variable $X$ modeled by the CIR process

$$
d X_{t}=\kappa\left(\theta-X_{t}\right) d t+\sigma \sqrt{X_{t}} d W_{t}, X_{0}=1,
$$

for $\theta, \kappa, \sigma>0$ and $\kappa \theta \geq \sigma^{2}$. As the Feller condition is satisfied, the process stays positive. The state space of $X$ is $I=(0, \infty)$. We assume that the short rate is $r\left(X_{t}\right)=X_{t}$ at time $t \geq 0$. Both the endpoints are LP, as shown in Appendix B. Equation (9) becomes

$$
\frac{1}{2} \sigma^{2} x h^{\prime \prime}(x)+\kappa(\theta-x) h^{\prime}(x)-x h(x)=-\lambda h(x) .
$$

Define

$$
m=\frac{\sqrt{\kappa^{2}+2 \sigma^{2}}-\kappa}{\sigma^{2}} .
$$

Then, it can be shown that $h_{0}(x)=e^{-m(x-1)}$ is a unique positive eigenfunction (up to constant multiples) and that its eigenvalue is $\lambda_{0}=m \kappa \theta$. The reciprocal of the pricing kernel is

$$
L_{t}=e^{m \kappa \theta t-m\left(X_{t}-1\right)}, t \geq 0,
$$

and the state variable follows

$$
d X_{t}=\kappa\left(\theta-\sqrt{1+2 \sigma^{2} / \kappa^{2}} X_{t}\right) d t+\sigma \sqrt{X_{t}} d B_{t}, X_{0}=1
$$


under the physical measure. This implies that, if the state variable is the CIR short rate model under the risk-neutral measure, then the state variable is still a CIR model with new parameters under the physical measure.

\subsubsection{CIR Process II}

Consider the state variable $X$ modeled as the CIR process in Equation (15) for $\theta, \kappa, \sigma>0$ and $\kappa \theta \geq \sigma^{2}$. The state space of $X$ is $I=(0, \infty)$. Assume that the short rate is $r\left(X_{t}\right)=\frac{k \theta}{X_{t}}$ at time $t \geq 0$. Then, Equation (9) becomes

$$
\frac{1}{2} \sigma^{2} x h^{\prime \prime}(x)+\kappa(\theta-x) h^{\prime}(x)-\frac{\kappa \theta}{x} h(x)=-\lambda h(x) .
$$

Both endpoints 0 and $\infty$ are LP by Appendix C. Then, the function $h_{0}(x)=x$ is the unique positive eigenfunction (up to constant multiples) and its eigenvalue is $\lambda_{0}=\kappa$. The reciprocal of the pricing kernel is

$$
L_{t}=e^{\kappa t} X_{t}, t \geq 0
$$

and the state variable follows

$$
d X_{t}=\kappa\left(\theta+\frac{\sigma^{2}}{\kappa}-X_{t}\right) d t+\sigma \sqrt{X_{t}} d B_{t}, X_{0}=1
$$

under the physical measure. The dynamics of the short rate $r_{t}=r\left(X_{t}\right), t \geq 0$ are

$$
\begin{aligned}
d r_{t} & =\left(\left(\kappa-\left(1-\frac{\sigma^{2}}{\kappa \theta}\right) r_{t}\right) r_{t} d t-\frac{\sigma}{\sqrt{\kappa \theta}} r_{t}^{3 / 2} d W_{t}\right. \\
& =\left(\kappa-r_{t}\right) r_{t} d t-\frac{\sigma}{\sqrt{\kappa \theta}} r_{t}^{3 / 2} d B_{t} .
\end{aligned}
$$

This implies that the short rate is a $3 / 2$ model under both the risk-neutral measure and the physical measure. This $3 / 2$ interest rate model has been studied in [17].

Remark 3. This model originates from the Laguerre equation. Refer to Appendix $C$ for more details.

\subsubsection{CEV Process}

Consider the state variable $X$ modeled by the CEV process

$$
d X_{t}=\mu X_{t} d t+\sigma X_{t}^{\beta+1} d W_{t}, X_{0}=1,
$$

for $\mu, \sigma>0$ and $\beta>0$. The state space of $X$ is $I=(0, \infty)$ ([18] Section 16.1.2). Assume that the short rate function is $r(x)=r_{0} x^{-2 \beta}$ for a positive constant $r_{0}$. Then, Equation (9) becomes

$$
\frac{1}{2} \sigma^{2} x^{2+2 \beta} h^{\prime \prime}(x)+\mu x h^{\prime}(x)-r_{0} x^{-2 \beta} h(x)=-\lambda h(x) .
$$

Both the endpoints 0 and $\infty$ are LP by Proposition 1. The function $h_{0}(x)=x e^{l\left(x^{-2 \beta}-1\right)}$ is a unique positive eigenfunction (up to constant multiples) and its eigenvalue is $\lambda_{0}=l \sigma^{2} \beta(1-2 \beta)-\mu$, where

$$
l:=\frac{\mu-\sqrt{\mu^{2}+2 \sigma^{2} r_{0}}}{2 \sigma^{2} \beta} .
$$

The reciprocal of the pricing kernel is

$$
L_{t}=X_{t} e^{\lambda_{0} t+l\left(X_{t}^{-2 \beta}-1\right)}, t \geq 0,
$$


and the state variable follows

$$
d X_{t}=\left(\mu-2 l \beta \sigma^{2}+\sigma^{2} X_{t}^{2 \beta}\right) X_{t} d t+\sigma X_{t}^{\beta+1} d B_{t}, X_{0}=1
$$

under the physical measure. The short rate $r_{t}=r\left(X_{t}\right), t \geq 0$ satisfies

$$
\begin{aligned}
d r_{t} & =\beta\left((2 \beta+1) \sigma^{2} r_{0}-2 \mu r_{t}\right) d t-2 \beta \sigma \sqrt{r_{0} r_{t}} d W_{t} \\
& =\beta\left((2 \beta-1) \sigma^{2} r_{0}-2 \sqrt{\mu^{2}+2 \sigma^{2} r_{0}} r_{t}\right) d t-2 \beta \sigma \sqrt{r_{0} r_{t}} d B_{t} .
\end{aligned}
$$

This implies that the short rate is a CIR process under both the risk-neutral measure and the physical measure.

\section{Impossibility of Recovery}

In this section, we argue that, in general, recovery is impossible. The integrability condition $h \in L_{w}^{2}(I)$ is essential for the uniqueness of the positive eigenfunction in the methods of Carr and $\mathrm{Yu}$ [2] and in Theorem 3. If this integrability condition is violated, then uniqueness does not hold.

\subsection{Integrability Condition}

We explore the following model as an example which has non-unique positive eigenfunctions. This model was also investigated by Carr and Yu [2]. Consider the state variable $X$ given by

$$
d X_{t}=\cot \left(X_{t}\right) d t+\sqrt{2} d W_{t}, X_{0}=\xi
$$

for $\xi \in(0, \pi)$. The range of $X$ is $I=(0, \pi)$. The short interest rate is assumed to be $r\left(X_{t}\right)=\csc ^{2}\left(X_{t}\right), t \geq$ 0 . Equation (9) becomes

$$
h^{\prime \prime}(x)+\cot (x) h^{\prime}(x)-\csc ^{2}(x) h(x)=-\lambda h(x)
$$

on $I$. This equation is singular and both the endpoints are LP. The eigenvalues are of the form $\lambda=$ $l(l+1)$ for $l>1$. The eigenfunctions of these eigenvalues are the associated Legendre polynomials $P_{l}(z)$, evaluated at $z=\cos (x)$. Among them, $P_{1}(\cos (x))=\sin (x)$ is the only positive eigenfunction with eigenvalue $\lambda=2$. The reciprocal of the pricing kernel is

$$
L_{t}=e^{2 t} \frac{\sin \left(X_{t}\right)}{\sin \left(X_{0}\right)}, t \geq 0,
$$

and the state variable follows

$$
d X_{t}=3 \cot \left(X_{t}\right) d t+\sqrt{2} d B_{t}
$$

under the physical measure.

However, we can find another solution without the integrability condition. For example, the function $h(x)=\frac{1}{\sin (x)}$ is also an eigenfunction, with eigenvalue $\lambda=0$. The reciprocal of the pricing kernel is

$$
L_{t}=\frac{\sin \left(X_{0}\right)}{\sin \left(X_{t}\right)}, t \geq 0
$$

and the state variable follows

$$
d X_{t}=-\cot \left(X_{t}\right) d t+\sqrt{2} d B_{t}
$$

under the physical measure. Without the integrability condition, in fact, Equation (19) has an infinite number of positive eigenfunctions (Theorem 4). 
The same issue arises in the original analysis of Ross. The set of possible states of the economy is denoted as $\mathcal{S}$. Ross assumes that $\mathcal{S}$ is finite. We believe that this assumption is unrealistic. Instead, we can consider a countable-state space: With the assumption that $\mathcal{S}=\mathbb{Z}$, the state variable can be equal to any number in $\mathbb{Z}$. In this case, the uniqueness theorem for the positive eigenvalue and the eigenvector with positive components requires some integrability conditions. As an example, let $a_{k}=2^{-k^{2}-2}$ and consider the following infinite-dimensional matrix $P=\left(p_{i j}\right)_{i \in \mathbb{Z}, j \in \mathbb{Z}}$ defined by $p_{i j}=a_{j-i}$.

$$
P=\left(\begin{array}{ccccc}
\ddots & & \vdots & & \ddots \\
& a_{0} & a_{1} & a_{2} & \\
\cdots & a_{-1} & a_{0} & a_{1} & \cdots \\
& a_{-2} & a_{-1} & a_{0} & \\
\therefore & & \vdots & & \ddots
\end{array}\right) .
$$

We fix an arbitrary $z>0$ and define $\delta=\sum_{k=-\infty}^{\infty} a_{k} z^{k}$. Then, a vector $\pi$ defined by $\pi=\left(z^{k}\right)_{k \in \mathbb{Z}}$ is an eigenvector of $P$, and the associated eigenvalue is $\delta$ :

$$
P \pi=\delta \pi
$$

As $z$ can be an arbitrary positive number, the uniqueness theorem of the Perron-Frobenius theorem does not hold. In this case, if we restrict our attention to $\pi \in l^{2}(\mathbb{Z})$ or $\pi \in\{$ bounded sequences on $\mathbb{Z}\}$, then $\pi=(1)_{k \in \mathbb{Z}}$ is a unique eigenvector (up to constant multiples) with positive components. In this analysis, $a_{k}=2^{-k^{2}-2}$ is just one of the possible examples. Any other sequence $\left\{a_{k}\right\}_{k \in \mathbb{Z}}$ can be chosen if $\sum_{k=-\infty}^{\infty} a_{k} \leq 1$ and $\sum_{k=-\infty}^{\infty} a_{k} z^{k}<\infty$ for an arbitrary $z$. For instance, $a_{-1}=\frac{1}{4}, a_{0}=\frac{1}{2}, a_{1}=\frac{1}{4}$, and $a_{k}=0$ for $k \neq-1,0,1$.

\subsection{Non-Uniqueness of Recovery}

As described previously, a physical measure cannot be uniquely determined from a risk-neutral measure without employing the integrability condition. Indeed, for any eigenpair $(\lambda, h)$ with $h(\cdot)>0$, the recovered measure with respect to $(\lambda, h)$ can be a candidate for a physical measure. In fact, there is no financial or economic rationale to believe that $h$ lies in $L_{w}^{2}(I)$. Without the integrability condition, the approach categorically fails to recover the physical measure.

Theorem 4. Suppose $\mathcal{L} h=-\lambda h$ has a positive solution $h$ for some $\lambda \in \mathbb{R}$. Then, for any $\hat{\lambda}$ with $\hat{\lambda}<\lambda$, $\mathcal{L} \hat{h}=-\hat{\lambda} \hat{h}$ has a positive solution $\hat{h}$.

The proof can be obtained by using the following theorem.

Theorem 5. (Bôcher) The differential equation

$$
u^{\prime \prime}(x)+p(x) u(x)=0
$$

has a positive solution $u$ on a non-trivial interval (possibly unbounded) I if and only if there is a $C^{1}$ solution $w(\cdot)$ on I of

$$
w^{\prime}(x)+w^{2}(x)+p(x) \leq 0 .
$$

See Bôcher [19] and Eliason and White [20] for the proof. A solution $h$ of $\mathcal{L} h=-\lambda h$ can be expressed as $h=u q$, where $q(x)=e^{-\int_{0}^{x} \frac{k(y)}{\sigma^{2}(y)} d y}$ and $u$ is a solution of

$$
u^{\prime \prime}(x)+\left(-\frac{d}{d x}\left(\frac{k(x)}{\sigma^{2}(x)}\right)-\frac{k^{2}(x)}{\sigma^{4}(x)}+\frac{2(-r(x)+\lambda)}{\sigma^{2}(x)}\right) u(x)=0 .
$$


By setting

$$
p(x)=-\frac{d}{d x}\left(\frac{k(x)}{\sigma^{2}(x)}\right)-\frac{k^{2}(x)}{\sigma^{4}(x)}+\frac{2(-r(x)+\lambda)}{\sigma^{2}(x)}
$$

and applying the Bôcher theorem, we obtain Theorem 4.

\section{Conclusions}

In this study, we reviewed the approaches of Ross [1] and Carr and Yu [2] for recovering a physical measure from a risk-neutral measure. Ross assumed a discrete-time and finite-state space and employed the concept of the utility of a representative agent. Carr and Yu developed the Ross recovery theory to a continuous-time setting with the martingale concept. They proposed an alternative, utility-free means of arriving at the same financial conclusion. The key assumption was that the reciprocal of the pricing kernel is transition-independent.

Carr and Yu demonstrated that the reciprocal of the pricing kernel is determined by a function $h$ which satisfies the SL Equation (12). We proved that, under the assumption that $h$ is in $L_{w}^{2}(I)$, recovery is feasible if and only if the SL equation has both LP endpoints. In addition, explicit recovery formulas were provided for several examples. In a general setting, recovery is not feasible as, without the integrability condition $h \in L_{w}^{2}(I)$, there are infinitely many positive eigenfunctions. Thus, a physical measure cannot uniquely determined from a risk-neutral measure in general.

Author Contributions: Conceptualization, H.P.; Formal analysis, S.A. and H.P.; Investigation, S.A. and H.P.; Supervision, H.P. All authors have read and agreed to the published version of the manuscript.

Funding: This work was supported by Research Resettlement Fund for the new faculty of Seoul National University. In addition, this research was supported by the National Research Foundation of Korea (NRF) grants funded by the Ministry of Science and ICT (No. 2017R1A5A1015626 and No. 2018R1C1B5085491) and the Ministry of Education (No. 2019R1A6A1A10073437) through Basic Science Research Program.

Conflicts of Interest: The authors declare no conflict of interest.

\section{Appendix A. Perron-Frobenius Theorem}

The Perron-Frobenius theorem (refer to Gantmakher [21]) is a mathematical preliminary that allows us to derive the Ross recovery theorem. Suppose that a matrix $P=\left(p_{i j}\right)$ has positive entries; that is, $p_{i j}>0$. Then,

(i) There exists a positive real number $\delta$ such that $\delta$ is an eigenvalue of $P$ and any other eigenvalue is strictly smaller than $\delta$ in absolute value.

(ii) The eigenspace associated with $\delta$ is one-dimensional.

(iii) There exists an eigenvector $\pi$ of $P$ with eigenvalue $\delta$ such that all the components of $\pi$ are positive.

(iv) All other eigenvectors must have at least one negative or non-real component.

(v) $\delta$ satisfies the inequalities

$$
\min _{i} \sum_{j} p_{i j} \leq \delta \leq \max _{i} \sum_{j} p_{i j}
$$

(vi) If $\sum_{j} p_{i j}<1$, then $\delta<1$.

\section{Appendix B. CIR Process I}

This section shows that both the endpoints are LP in the model in Section 4.3.3. Recall Equation (16):

$$
\frac{1}{2} \sigma^{2} x h^{\prime \prime}(x)+\kappa(\theta-x) h^{\prime}(x)-x h(x)=-\lambda h(x) .
$$


Unfortunately, Proposition 1 cannot be applied, as the weight function $w \in L^{1}(c, \infty)$ for any $c>0$ where

$$
w(x)=\frac{2}{\sigma^{2}} x^{\frac{2 \kappa \theta}{\sigma^{2}}-1} e^{-\frac{2 \kappa x}{\sigma^{2}}} .
$$

Instead, we use the definition of the LP/LC points stated in Section 4.1. One can show that

$$
\bar{h}(x)=e^{\frac{\kappa+\sqrt{\kappa^{2}+2 \sigma^{2}}}{\sigma^{2}} x} x^{1-\frac{2 \kappa \theta}{\sigma^{2}}}
$$

is an eigenfunction (this can be obtained by trying an ansatz $\left.h(x)=e^{m x} x^{l}\right)$. For $\kappa \theta \geq \sigma^{2}$, the function $\bar{h}$ is neither in $L_{w}^{2}((0,1))$ nor in $L_{w}^{2}((1, \infty))$. Thus, both the endpoints 0 and $\infty$ are LP. Note that, if $\kappa \theta<\sigma^{2}$, then the endpoint $\infty$ is LP, but the endpoint 0 is LC; thus, there are infinitely many positive eigenfunctions.

\section{Appendix C. CIR Process II}

This section shows that both the endpoints are LP in the model in Section 4.3.4. For simplicity, we set $\sigma=\sqrt{2}$, which does not alter the LC/LP classification of the endpoints. Recall Equation (17):

$$
x h^{\prime \prime}(x)+\kappa(\theta-x) h^{\prime}(x)-\frac{\kappa \theta}{x} h(x)=-\lambda h(x) .
$$

The weight function $w$ is

$$
w(x)=x^{\kappa \theta-1} e^{-\kappa x} .
$$

It is enough to show that there exists an eigenfunction that is neither in $L_{w}^{2}((0,1))$ nor in $L_{w}^{2}((1, \infty))$. This can be proved by considering the eigenfunction $h(x)=x \int_{1}^{x} z^{-\kappa \theta-2} e^{\kappa z} d z$ with eigenvalue $\lambda=\kappa$.

For the rest of this section, we discuss how the authors formulated this model. Originally, Equation (A1) is derived from the Laguerre equation

$$
-x y^{\prime \prime}(x)-(\alpha+1-x) y^{\prime}(x)=l y(x) \text { for } 0<x<\infty .
$$

This Laguerre equation is LP at $\infty$ for all $\alpha \in(-\infty, \infty)$ and LP at 0 for $\alpha \geq 1$ and $\alpha \leq 1$; see ([22] Section 27) for more details. It is well-known that, for $\alpha>-1$, the classical Laguerre polynomials are eigenfunctions and the eigenvalues are $l_{n}=n$ for $n=0,1,2, \cdots$, which are independent of $\alpha$. For $l=0$, we can directly compute two linearly independent solutions

$$
y_{1}(x)=1, \quad y_{2}(x)=\int_{1}^{x} z^{-\alpha-1} e^{z} d z .
$$

Now, we transform the Laguerre equation to derive (A1). We change the first-order term $-(\alpha+1-x) y^{\prime}(x)$ of the Laguerre equation to $-(\alpha+1-\kappa x) y^{\prime}(x)$ :

$$
-x y^{\prime \prime}(x)-(\alpha+1-\kappa x) y^{\prime}(x)=\operatorname{ly}(x) .
$$

By substituting

$$
y(x)=\frac{h(x)}{x}, \quad \alpha=\kappa \theta+1, \quad l=\lambda-\kappa,
$$

we obtain Equation (A1).

\section{References}

1. Ross, S. The recovery theorem. J. Financ. 2015, 70, 615-648. [CrossRef]

2. Carr, P.; Yu, J. Risk, return, and Ross recovery. J. Deriv. 2012, 20, 38-59. [CrossRef]

3. Borovička, J.; Hansen, L.P.; Scheinkman, J.A. Misspecified recovery. J. Financ. 2016, 71, 2493-2544. [CrossRef]

4. Jensen, C.S.; Lando, D.; Pedersen, L.H. Generalized recovery. J. Financ. Econ. 2019, 133, 154-174. [CrossRef] 
5. Park, H. Ross recovery with recurrent and transient processes. Quant. Financ. 2016, 16, 667-676. [CrossRef]

6. Qin, L.; Linetsky, V. Positive eigenfunctions of Markovian pricing operators: Hansen-Scheinkman factorization, Ross recovery, and long-term pricing. Oper. Res. 2016, 64, 99-117. [CrossRef]

7. Walden, J. Recovery with unbounded diffusion processes. Rev. Financ. 2017, 21, 1403-1444. [CrossRef]

8. Audrino, F.; Huitema, R.; Ludwig, M. An empirical analysis of the Ross recovery theorem. SSRN 2015. Available online: https:/ / papers.ssrn.com/sol3/papers.cfm?abstract_id=2433170 (accessed on 9 April 2020). [CrossRef]

9. Bakshi, G.; Chabi-Yo, F.; Gao, X. A recovery that we can trust? Deducing and testing the restrictions of the recovery theorem. Rev. Financ. Stud. 2018, 31, 532-555. [CrossRef]

10. Dillschneider, Y.; Maurer, R. Functional Ross recovery: Theoretical results and empirical tests. J. Econ. Dyn. Control 2019, 108, 103750. [CrossRef]

11. Flint, E.; Maré, E. Estimating option-implied distributions in illiquid markets and implementing the Ross recovery theorem. S. Afr. Actuar. J. 2017, 17, 1-28.

12. Kiriu, T.; Hibiki, N. Estimating forward looking distribution with the Ross recovery theorem. J. Oper. Res. Soc. Jpn. 2019, 62, 83-107. [CrossRef]

13. Barucci, E.; Fontana, C. Financial Markets Theory: Equilibrium, Efficiency and Information; Springer: Berlin/Heidelberg, Germany, 2017.

14. Pinsky, R. G. Positive Harmonic Function and Diffusion; Cambridge University Press: Cambridge, UK, 1995.

15. Zettl, A. Sturm-Liouville Theory; American Mathematical Soc.: Providence, RI, USA, 2010.

16. Fulton, C.T.; Pruess, S.; Xie, Y. The automatic classification of Sturm-Liouville problems. J. Appl. Math. Comput. 2005, 124, 149-186.

17. Ahn, D.H.; Gao, B. A parametric nonlinear model of term structure dynamics. Rev. Financ. Stud. 1999, 12, 721-762. [CrossRef]

18. Campolieti, G.; Makarov, R.N. Financial Mathematics: A Comprehensive Treatment; Chapman and Hall/CRC: Boca Raton, FL, USA, 2018.

19. Bôcher, M. Non-oscillatory linear differential equations of the second order. Bull. Am. Math. Soc. 1901, 7, 333-340. [CrossRef]

20. Eliason, S.B.; White, L.W. On positive solutions of second order elliptic partial differential equations. Hiroshima Math. J. 1982, 12, 469-484. [CrossRef]

21. Gantmakher, F.R. The Theory of Matrices; American Mathematical Soc.: Providence, RI, USA, 1959.

22. Amrein, W.O.; Hinz, A.M.; Pearson, D.B. Sturm-Liouville Theory: Past and Present; Springer Science \& Business Media: Berlin/Heidelberg, Germany, 2005. 